\title{
Reallabore als Rahmen transformativer und transdisziplinärer Forschung: Ziele und Designprinzipien
}

Richard Beecroft, Helena Trenks, Regina Rhodius, Christina Benighaus \& Oliver Parodi

\section{$1 \quad$ Einleitung}

$\mathrm{Zu}$ Reallaboren, ihrer Definition und ihrem Aufbau, ihren Zielen und Methoden besteht inzwischen ein lebendiger und teils divergierender Diskurs. Die Kontroversen beruhen nicht nur darauf, dass sie ein noch junges, wenig beschriebenes Format sind, sondern auch auf ihrem umfassenden Anspruch: Sie schließen eine Lücke zwischen Forschung und Praxis; sie arbeiten transdisziplinär und transformativ, indem sie wissenschaftliche Forschung mit Beiträgen zu einem gesellschaftlichen Wandel kombinieren.

Erste Definitionen von Reallaboren stellten den gesellschaftlichen Bedarf nach „wissenschaftsgeleitete[r] Unterstützung des Transformationsprozesses im Sinne der Transdisziplinarität" heraus (MWK 2013, S. 31; s. auch Wagner und Grunwald 2015; Arnold und Piontek 2018), auch weil zu diesem Zeitpunkt noch kaum Projekte unter diesem Begriff firmierten. In einer zweiten Phase, in der eine Vielzahl von Reallaboren die Arbeit aufnahm, lag der Schwerpunkt auf der Diskussion von Charakteristika und Kriterien, die helfen zu entscheiden, was berechtigterweise als Reallabor bezeichnet wird. Beecroft und Parodi (2016) listen zum Beispiel als Kriterien „Forschungsorientierung, normative Orientierung an Nachhaltigkeit, Transdisziplinarität, Transformativität, zivilgesellschaftliche Orientierung, Langfristigkeit und Laborcharakter" auf. Defila und Di Giulio (2018) betonen in der Einführung zu diesem Buch als zwingende Anforderung an Reallabore (nebst der Transdisziplinarität), dass diese ein gesellschaftlich legitimiertes Ziel verfolgen müssen, das ethisch gut begründet und gemeinwohlorientiert ist.

An diese Definitionen anknüpfend soll hier ein Verständnis von Reallaboren vorgelegt werden, das auf der Reflexion praktischer Erfahrungen basiert, und das da- 
$\mathrm{zu}$ geeignet ist, methodologische ${ }^{1}$ Entscheidungen abzuleiten - etwa die in diesem Beitrag skizzierten Designprinzipien. Ausgangspunkt hierfür ist ein Verständnis von Reallaboren als Rahmen für transdisziplinäre Projekte. ${ }^{2}$ Diese können $\mathrm{zu}$ unterschiedlichen Zeitpunkten im Labor starten und enden, unterschiedliche Akteure aus Wissenschaft und Praxis einbinden und sich in den Fragestellungen unterscheiden. Die charakteristische Form transdisziplinärer Projekte in einem Reallabor sind Realexperimente ${ }^{3}$, bei denen in einem kooperativen Prozess zwischen Akteuren aus der Wissenschaft und aus der Praxis Experimente geplant, durchgeführt und ausgewertet werden (Co-Design und Co-Produktion). Transdisziplinäre Projekte initiieren und begleiten reale Transformationsprozesse (insbesondere in Form von Interventionen) und produzieren Wissen über solche Prozesse. In transdisziplinären Projekten kann eine Vielzahl von Methoden und Methodenkombinationen zum Einsatz kommen, darunter auch solche, die in einem Reallabor neu entwickelt werden ${ }^{4}$. Ein Reallabor entfaltet sein Potential gerade dann, wenn es Erfahrungsaustausch und Synergien zwischen parallelen und/oder aufeinander folgenden Projekten ermöglicht. ${ }^{5}$ Abb. 1 zeigt beispielhaft das Design des Reallabors 131: KIT findet Stadt (BaWü-Lab R131) (s. a. Steckbrief im Anhang dieses Buchs), in dem transdisziplinäre Projekte eingebettet waren in eine transdisziplinäre Infrastruktur und in dem übergreifende Aufgaben definiert wurden, um diese Projekte zu unterstützen.

1 Methodologie wird im Kontext dieses Beitrags verwendet als die Auswahl, Entwicklung, Bewertung und Kombination von Methoden, die in den transdisziplinären Projekten in einem Reallabor zum Einsatz kommen. Die Methoden entstammen nicht zwangsläufig der Forschung (z. B. Mediation).

2 Auch naturwissenschaftliche und technische Labore stellen einen Rahmen dar, um gestaltbare Bedingungen, Beobachtbarkeit und Sicherheit von Experimenten unter Anwendung spezifischer Methoden zu ermöglichen.

3 Unter Realexperimenten verstehen die Autor(inn)en hier Interventionen, die gemeinsam mit Akteuren aus der Praxis konzipiert und durchgeführt werden, und die im Rahmen eines Reallabors hinreichend gut beeinflusst und wissenschaftlich begleitet werden können (Groß et al. 2005; Parodi et al. 2016b). Da der Begriff in Praxiskontexten leicht missverstanden werden kann, werden Realexperimente in der Umsetzung oft anders bezeichnet, z. B. als Nachhaltigkeitsexperimente (Trenks et al. 2018). In diesem Text werden Realexperimente unter dem Begriff transdisziplinäre Projekte subsumiert.

4 Dieses Buch stellt eine erste systematische Sammlung und Darstellung dar von Methoden, die in Reallaboren - und zwar solchen, die im Rahmen der beiden Förderlinien „Reallabore“ und „Reallabore Stadt“ in Baden-Württemberg ab 2015 gefördert wurden (nachstehend als ,BaWü-Labs“ bezeichnet) - neu entwickelt bzw. angepasst oder kombiniert wurden.

5 Die hier zugrunde gelegte Architektur greift in Reallaboren nicht immer, so werden Reallabore z. B. selbst als Methode der Intervention in Wissensräume diskutiert (wie z. B. das BaWü-Lab Urban Office - Nachhaltige Stadtentwicklung in der Wissensgesellschaft (s. Steckbrief im Anhang dieses Buchs), s. Marquardt und West 2016). 


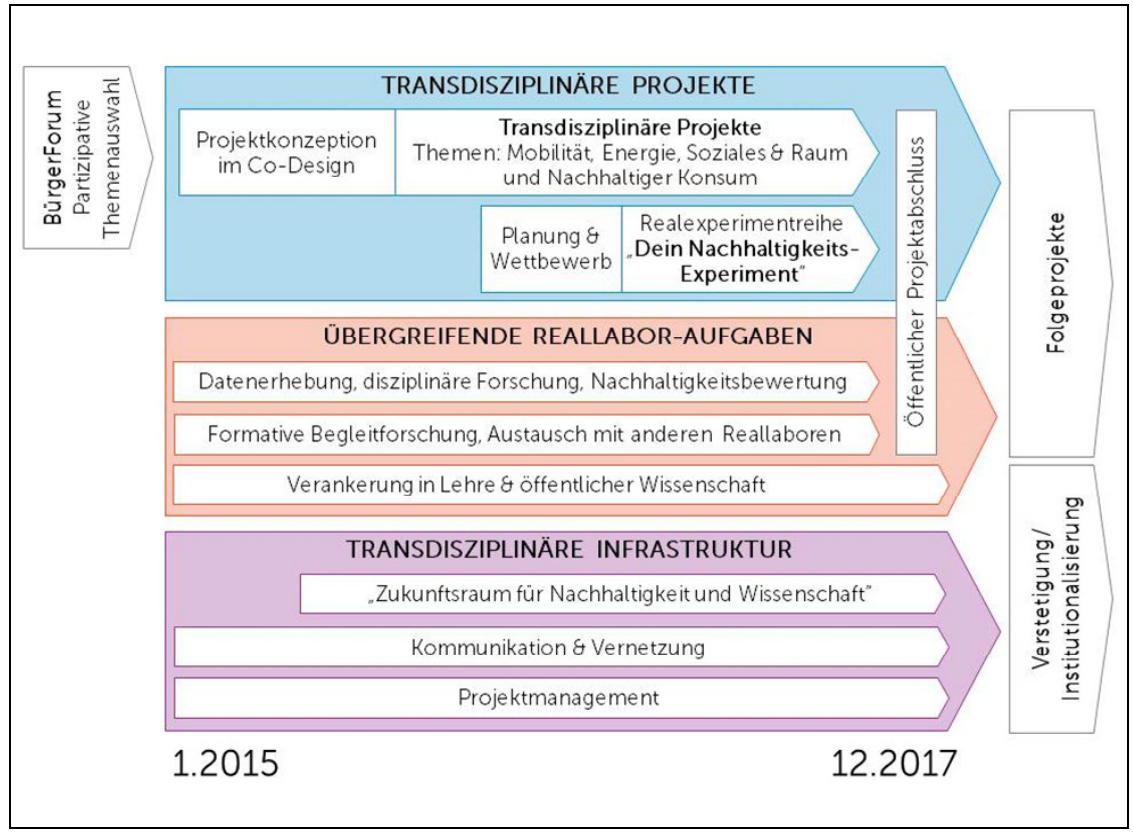

Abbildung 1: Das Design des BaWü-Labs R131 in Karlsruhe, in dem die hier dargestellte Architektur gewählt wurde. C) R131.

Reallabore unterstützen transdisziplinäre Projekte in zweifacher Hinsicht:

1) indem sie einen übergreifenden Zielhorizont eröffnen, in den sich Einzelaktivitäten einordnen lassen.

2) indem in ihrem Forschungsdesign Rahmenentscheidungen (etwa zu Arten der Datensammlung oder zu Evaluationsstrategien) getroffen werden, durch die die einzelnen transdisziplinären Projekte aufeinander abgestimmt werden können.

Im Hinblick auf die transdisziplinäre Forschung ist am Reallabor-Ansatz neu, dass transdisziplinäre Projekte nicht für sich stehen, sondern dass mit einem Reallabor ein spezifischer, ebenfalls transdisziplinärer Rahmen für sie etabliert wird. Dieser bietet eine geeignete Infrastruktur und erlaubt, übergreifende transdisziplinäre Aufgaben (z. B. Monitoring neuer Themen, Integration von Ergebnissen zwischen transdisziplinären Projekten, Aufbau langfristiger Kooperationen) zu bearbeiten, die ansonsten leicht zu kurz kommen.

Der Begriff „Reallabor“ entstammt den Debatten um eine transdisziplinäre und transformative Nachhaltigkeitsforschung (WBGU 2011; Schneidewind und Sin- 
ger-Brodowski 2013; 2015). Insbesondere die vierzehn ab 2015 in Baden-Württemberg eingerichteten BaWü-Labs folgen dem Leitbild einer „Wissenschaft für Nachhaltigkeit"“. ${ }^{6}$ Andere legitimierte, ethisch gut begründete und gemeinwohlorientierte gesellschaftliche Ziele ließen sich in ähnlicher Weise in Reallaboren bearbeiten (etwa Inklusion im Bildungsbereich), mit demselben Anspruch, Transformationsprozesse anzustoßen, zu begleiten und zu beforschen.

Für den Kontext dieses Buches legen die Autor(inn)en dementsprechend folgendes Verständnis von Reallaboren zugrunde:

Reallabore sind Einrichtungen an der Schnittstelle von Wissenschaft und Praxis. Sie bieten einen Rahmen, um Forschungs-, Praxis- und Bildungsziele zu verfolgen. Reallabore sind transformativ ausgerichtet und verfolgen gesellschaftlich legitimierte, ethisch gut begründete und gemeinwohlorientierte Ziele. Designprinzipien für Reallabore dienen der sachlichen, räumlichen und zeitlichen Rahmung und dem Aufbau einer Rollenkonstellation beteiligter Akteure, die den zu bearbeitenden Transformationsprozessen angemessen sind. In Reallaboren werden transdisziplinäre Projekte (insbesondere Realexperimente) umgesetzt. Diese Projekte werden im Sinne einer experimentellen und reflexiven Arbeitsweise kontinuierlich reflektiert und ihr Projektverlauf wird dementsprechend angepasst.

Der folgende Text führt die in dieser Definition angelegte Gliederung in Ziele und Designprinzipien aus. In Kapitel 2 wird der Zielhorizont eines Reallabors dargestellt. Die BaWü-Labs, aus deren Begleitforschung dieses Buch hervorgegangen ist, verstehen sich als ,transformative Forschung“ (WBGU 2011; Schneidewind und Singer-Brodowski 2013). Als solche möchten sie gleichermaßen reale Transformationsprozesse anstoßen (Praxisziele) und - soweit möglich verallgemeinerbares - Wissen über Transformation hervorbringen (Forschungsziele). Neben diesen beiden etablierten Zieldimensionen sehen die Autor(inn)en in der Ermöglichung von Lernen eine dritte, eigenständige Zieldimension (Bildungsziele), ohne die Transformation nicht in einem umfassenden Sinne denkbar ist. Kapitel 3 beschreibt zentrale Prinzipien für das Design von Reallaboren: Die Festlegung eines angemessenen thematischen, räumlichen und zeitlichen Rahmens, die angemessene Gestaltung der Akteursrollen sowie den zu etablierenden experimentell-reflexiven Forschungsstil, der auch ein Reallabor als Ganzes lernfähig hält. Den Schluss in Kapitel 4 bildet ein Schema, basierend auf den dargestellten Zielen und Designprinzipien, das als Reflexionsrahmen und Evaluationsinstrument für das Design eines konkreten Reallabors dienen kann. In diesem Schema sind Lessons Learnt aus den BaWü-Labs zusammengestellt.

6 Siehe auch: https://mwk.baden-wuerttemberg.de/de/forschung/forschungspolitik/ wissenschaft-fuer-nachhaltigkeit/ (zugegriffen am 12.01.2018). 


\section{Ziele der Reallaborarbeit}

Warum sollte man ein Reallabor aufbauen, wenn man die Energie doch auch gänzlich in die transdisziplinären Projekte stecken könnte? Die Rechtfertigung für den Aufbau eines Reallabors als Rahmen liegt darin, dass die verfolgten Ziele besser mit als ohne Reallabor zu erreichen sind. Hierbei gilt es drei Zieldimensionen zu berücksichtigen - Forschungs-, Praxis- und Bildungsziele - die in den folgenden Abschnitten (2.1-2.3) erläutert werden. Dabei nehmen die Bildungsziele insofern eine Sonderstellung ein, als zwar das Wechselspiel von Praxis- und Forschungszielen in der Literatur zu Transdisziplinarität und in verwandten Diskursen schon intensiv diskutiert wurde (z. B. Wagner und Grunwald 2015), aber Bildungsziele bislang wenig Berücksichtigung fanden.

\subsection{Forschungsziele im Reallabor}

Ein Reallabor dient im Hinblick auf die Forschungsziele dazu, das Erzeugen von Wissen zu unterstützen, Wissen zu sammeln und zu integrieren (für Methoden der Integration s. Bergmann et al. 2010) sowie seine Qualität zu bewerten. Dabei steht solches Wissen im Vordergrund, das aus Transformationsprozessen hervorgeht und für Transformationsprozesse relevant ist. ${ }^{7}$ Dieses Wissen lässt sich unterscheiden in erstens Systemwissen, zweitens Zielwissen, das die Richtung des angestrebten Transformationsprozesses auch über den Rahmen des Reallabors hinaus beschreibt, sowie drittens Transformationswissen, das die Möglichkeiten der Veränderungen beschreibt (zu diesen Wissensarten s. CASS und ProClim1997). Damit stellt ein Reallabor eine Form der transformativen Forschung dar, in der sowohl interdisziplinäre als auch transdisziplinäre Arbeitsweisen erforderlich sind.

Interdisziplinär gilt es, unterschiedliche disziplinäre Perspektiven zu integrieren, um den Gegenstand der Forschung möglichst umfassend beschreiben zu können. So kann auch der Anschluss der Reallaborforschung an disziplinäre Diskurse sichergestellt werden, z. B. durch den Bezug auf einschlägige Theorien und Befunde oder durch die Anwendung von disziplinären Standards bei der Datenerhebung. Sofern sich entsprechende Wissenslücken zeigen, können in einem Reallabor auch disziplinäre Projekte sinnvoll sein. Die umfassende, interdisziplinäre Beschreibung der Problemstellung, des Bezugsraums sowie der relevanten Akteure in einem Reallabor dient dazu, die übertragbaren Aspekte von fallspezifischen Aspekten unterscheiden zu können. Diese umfassende Darstellung der

7 Indirekt ist natürlich auch die Weiterentwicklung von Reallaboren in Theorie und Methodologie Teil der Forschungsziele. 
Spezifika eines Reallabors erlaubt es auch, durch ein Monitoring von Veränderungen unerwartete Effekte der transdisziplinären Projekte zu identifizieren.

Transdisziplinär gilt es, wissenschaftliche Perspektiven mit außerwissenschaftlichen anhand von exemplarischen Transformationsprozessen zu verbinden. Diese Integration sichert die gesellschaftliche Relevanz der bearbeiteten Forschungsfragen und dient der kritischen Auseinandersetzung mit dem Sinn und der Qualität der transdisziplinären Arbeit. Im Reallabor können Akteure aus der Praxis ${ }^{8}$ in unterschiedlichen Rollen einbezogen werden. Sie können mit darüber entscheiden, was experimentell neu erschlossen wird, welche Transformationsprozesse angestoßen oder bearbeitet werden, wie das Wissen integriert wird und wie mit normativen Fragen umgegangen werden soll (Finke und Laszlo 2014). Transdisziplinäre Arbeit muss hier die Reflexion der Akteure aus der Wissenschaft über ihre eigene Rolle im Reallabor und im transdisziplinären Projekt, ggf. aber auch als Akteure im Transformationsprozess miteinschließen (experimentellreflexive Arbeitsweise, s. Abschnitt 3.5).

\subsection{Praxisziele im Reallabor}

Im Hinblick auf die Praxisziele dient ein Reallabor dazu, Transformationsprozesse anzustoßen, zu fördern und mitzugestalten. Im Folgenden werden, basierend auf dem spezifischen Fall der BaWü-Labs, die auf Nachhaltigkeitstransformationen abzielen, fünf Praxisziele unterschieden:

1) Nachhaltigkeitstransformationen anstoßen und dabei unerwünschte Folgen erkennen und vermeiden (Transformationsziel)

2) Ergebnisse auf andere Transformationsprozesse übertragen (Transfer- und Upscalingziel)

3) Kultur der Nachhaltigkeit im Umfeld des Reallabors fördern (Kulturziel)

4) Kooperation der Akteure stabilisieren (Kooperationsziel)

5) Empowerment der Akteure aus der Praxis anstreben (Empowermentziel)

Die ersten beiden Ziele betreffen vornehmlich transdisziplinäre Projekte in einem Reallabor, die letzten drei betreffen gleichermaßen ein Reallabor als Ganzes.

Das erste Praxisziel besteht darin, konkrete Transformationsprozesse Richtung Nachhaltigkeit anzustoßen und zu begleiten. Diese können eine weite Spanne umfassen, etwa Veränderungen einer Infrastruktur, (mikro-)ökonomischer Struk-

8 Mit Akteuren aus der Praxis sind Personen aus Initiativen, Vereinen, Unternehmen, öffentlicher Verwaltung oder Politik gemeint, aber gleichermaßen auch engagierte Einzelpersonen, die in Bezug zum bearbeiteten Transformationsprozess stehen. 
turen oder des sozialen Zusammenlebens. Teil des ersten Praxisziels ist zudem die Vermeidung nicht-intendierter Folgen, auch außerhalb der thematischen, räumlichen und zeitlichen Grenzen des Reallabors.

Das zweite Praxisziel betrifft die Übertragung ${ }^{9}$ der Ergebnisse auf andere Räume (z. B. Regionen) oder gesellschaftliche Kontexte (Transfer) und eine gesellschaftliche und politische Anschlussfähigkeit der Ergebnisse für weiterreichende Entscheidungen (upscaling). ${ }^{10}$

Das dritte Praxisziel ist weitaus schwerer zu greifen, aber nicht minder wichtig: Indem ein Reallabor gestaltend und verändernd in seinem Kontext wirkt, kann es eine gelebte ,Kultur der Nachhaltigkeit' fördern (Krainer und Trattnigg 2017; Parodi 2015): Lebensweltliche ${ }^{11}$ Themen und Fragestellungen werden räumlich und zeitlich eingegrenzt, um sie zu bearbeiten. Ein Reallabor baut Brücken zwischen der spezifischen Praxis und dem abstrakten und globalen Verständnis Nachhaltiger Entwicklung. Es ermöglicht damit auch eine tiefergehende Identifikation mit Nachhaltiger Entwicklung und den Wandel von Handlungsweisen, Governancestrukturen und Lebensstilen. So kann auch ein Austausch zwischen sehr verschiedenen Nachhaltigkeitsperspektiven vorangetrieben werden (Grunwald 2016).

Das vierte Praxisziel besteht in der Herstellung einer vertrauensvollen, engen Kooperation der Akteure aus Wissenschaft und Praxis auf Augenhöhe (Di Giulio et al. 2016). Ein Reallabor als Teil einer demokratischen Gesellschaft sollte diese Art der Zusammenarbeit nicht nur als Mittel (z. B. zur Erhebung lokalen Wissens) nutzen, sondern muss sie selbst als Teil des Zielhorizontes integrieren. Dementsprechend gilt es, auch marginalisierte Gruppen, je nach thematischem Schwerpunkt, aktiv einzubeziehen. Bestenfalls sollte auch in der Art der Kooperation eine Kultur der Nachhaltigkeit zum Ausdruck kommen. Eine gelingende vertrauensvolle Kooperation der beteiligten Akteure aus Wissenschaft und Praxis ist zwar ein Praxisziel, stellt zugleich aber auch eine notwendige Grundlage für die Erreichung der anderen Forschungs-, Praxis- und Bildungsziele dar.

Das fünfte Praxisziel betrifft das Empowerment von Akteuren. Die Stufen der Partizipation in einem Reallabor, die sich in Anlehnung an Arnstein (1969) formulieren lassen, bauen oft aufeinander auf. Information und Konsultation stellen

9 Zumindest: Die Sicherung der Übertragbarkeit.

10 Es ist nach der Erfahrung in den BaWü-Labs wichtig, im Blick zu behalten, dass auch die Akteure aus der Praxis nicht nur, ihre' Praxis im Rahmen des Reallabors vor Augen haben, sondern oft auch schon weitreichende Transfer- und Upscalingziele mitbringen.

11 Lebenswelt bezeichnet in diesem Text nicht nur die Alltagswelt der Einzelnen, sondern auch alle Themen, die diese auf ggf. grundsätzliche Weise betreffen, etwa weitreichende politische Entscheidungen oder technologische Durchbrüche. 
dementsprechend die Voraussetzungen für eine intensivere Kooperation und letztlich für Empowerment her. ${ }^{12}$ Die BaWü-Labs betreiben Partizipation von Information und Konsultation über Kooperation bis zum Empowerment (Arnstein 1969; für Reallaborarbeit angepasst in Mayer-Soylu et al. 2016). Empowerment wird in einem Reallabor angestrebt, damit die Akteure - sowohl aus der Praxis wie aus der Wissenschaft - in die Lage versetzt werden, auch über die Laufzeit der transdisziplinären Projekte hinaus an den Transformationsprozessen langfristig aktiv teilhaben zu können.

Ein Reallabor, dessen thematischer Fokus sich nicht primär an Nachhaltiger Entwicklung orientiert, wird teils andere Praxisziele verfolgen. Es ist jedoch zu erwarten, dass sich eine ähnliche Spanne von Praxiszielen mit Bezug zu Transformation, Transfer, Kultur, Kooperation und Empowerment ausdifferenzieren lässt.

\subsection{Bildungsziele im Reallabor}

Die Transformationsprozesse, die von einem Reallabor angestoßen und begleitet werden, erfordern in aller Regel auch Veränderungen der Lebensweise von Einzelnen, Veränderungen von Rahmenbedingungen und Entscheidungsstrukturen auf allen Ebenen und Verschiebungen in gesellschaftlichen Wertesystemen. Hierzu bedarf es verbundener individueller und gesellschaftlicher Lernprozesse (Beecroft und Dusseldorp 2009; Beecroft und Dusseldorp 2012; Parodi et al. 2016b), die das Lernen nicht als nachgängige Verpflichtung, sondern als Teil der Transformationsprozesse auffassen.

Während Forschungs- und Praxisziele in der Regel explizit bearbeitet werden, bleiben die Bildungsziele im Reallabor bis dato oft implizit oder randständig (Singer-Brodowski et al. 2018). Es ist aber lohnend, Bildungsziele als Teil der Planung, Begleitung und Evaluation von transdisziplinären Projekten (und den darin eingesetzten Methoden) explizit mitzudenken. So lässt sich beispielsweise erheben, ob die Beteiligten nur eigene Bildungsziele verfolgen (und welche, z. B. Kompetenzentwicklung ${ }^{13}$, Wissenserwerb, Selbstentwicklung, Erleben), oder inwieweit auch die Absicht vorliegt, Andere weiterzubilden. Verschiedene beteiligte Gruppen und Personen haben ggf. sehr unterschiedliche Bildungsziele. Diese wiederum lassen sich aber keineswegs zwangsläufig aus ihrer Rolle ableiten, dass also z. B. Wissenschaftler(innen) nur aus ihren Realexperimenten lernen wollen, Studierende ausschließlich an formaler Bildung (,mit Schein') interessiert sind oder zivilgesellschaftliche Partner nur auf Erfahrung in ihrem Feld aus sind.

12 Der an dieser Stelle angelegte Partizipationsbegriff entstammt der Theorie politischer Partizipation, er eignet sich insbesondere bezogen auf die Praxisziele eines Reallabors.

13 Eine im Kontext von Nachhaltigkeitstransformationen gut anschlussfähige Systematik von Kompetenzen haben Wiek et al. (2011) vorgelegt. 
Ein Reallabor zielt dabei nicht darauf ab, selbst ein (formelles oder informelles) Bildungsangebot $\mathrm{zu}$ sein, sondern darauf, eine Lernumgebung zu sein (SingerBrodowski et al. 2018): Ein Reallabor bietet einen unterstützenden, geschützten Rahmen für Information, Austausch, Kooperation, Interventionen sowie Evaluation und Reflexion. Die Herstellung eines solchen ,Freiraums' ermöglicht Bildungsprozesse bei den beteiligten Akteuren, unabhängig davon, ob dies explizit mit dem Ziel der Bildung geschieht. Solche Prozesse lassen sich unterstützen, wenn bewusst Methoden aus dem Bildungskontext integriert werden und Bildung verbunden mit Praxis- und Forschungszielen verfolgt wird. Bildungsziele werden in der Regel auf der Ebene der einzelnen transdisziplinären Projekte verfolgt; zumindest die Evaluation, der Vergleich und die Erfahrungssammlung aus den einzelnen Realexperimenten stellen aber wichtige Bildungsprozesse auf Ebene eines Reallabors als Ganzes dar.

Bildungsziele sind unter verschiedenen Perspektiven als integraler Bestandteil jedes Reallabors zu sehen:

- Reallabore arbeiten gerade deshalb experimentell-reflexiv, weil ideale Transformationsprozesse (noch) nicht existieren und alle dahingehenden Versuche gründlich durchdacht werden müssen.

- Reallabore sind nicht statisch, sondern müssen selbst flexibel und lernfähig sein.

- Die Suche nach angemessenen Transformationswegen stellt einen gesellschaftlichen Lernprozess dar, zu dem Reallabore beitragen, d. h. Transformationsprozesse lassen sich in einem grundsätzlichen Sinn als gesellschaftliche Lernprozesse (Schneidewind und Singer-Brodowski 2015) auffassen.

\subsection{Komplexität des Zielhorizonts im Reallabor}

In einem Reallabor kann eine Vielzahl unterschiedlicher Akteure aus der Praxis (s. Eckart et al. 2018) und aus der Wissenschaft beteiligt sein, die alle jeweils eigene Ziele in ein Reallabor einbringen. Dabei sind drei Ebenen zu unterscheiden: Erstens die Ziele des Reallabors als Ganzes, zweitens die Ziele der transdisziplinären Projekte (bzw. Realexperimente) und drittens die individuellen Ziele. Die Abstimmung dieser drei Ebenen ist von zentraler Bedeutung. Dazu müssen alle beteiligten Akteure ihre Ziele auf Augenhöhe aushandeln und miteinander verbinden. Besonders zu beachten ist hierbei, dass sich die Ziele der Beteiligten im Projektverlauf konkretisieren können und dass diese in mehreren der drei Dimensionen liegen können: Ein und derselbe Akteur kann Forschungs-, Praxis- und Bildungsziele einbringen. 
Vor diesem Hintergrund muss in den transdisziplinären Projekten durchaus mit Konflikten gerechnet werden. Diese können entweder als Interessenkonflikte (zwischen Akteuren) oder als Zielkonflikte (zwischen den Zielen selbst) zu Tage treten (detailliert in Dusseldorp 2017). Das spezielle Potential eines Reallabors liegt darin, dass mit Ziel- und Interessenkonflikten auf Ebene der transdisziplinären Projekte aktiv umgegangen werden kann, z. B. indem im Reallabor Mediation angeboten wird oder Folgeprojekte anders ausgelegt werden.

\section{Designprinzipien für Reallabore}

Um sein volles Potential auszuschöpfen, benötigt ein Reallabor ein durchdachtes Forschungsdesign. Die fünf Designprinzipien, die im Folgenden aufgelistet werden, dienen dazu, mit einem Reallabor einen optimalen Rahmen für transdisziplinäre Arbeit herzustellen. Sie ergänzen allgemeine Designprinzipien aus Forschungs- und Praxiskontexten (etwa: Methoden auf Fragestellung abstimmen, Effizienz der Abläufe herstellen, Klärung von Verantwortlichkeiten), die hier nicht ausgeführt werden.

1) Problem- und Themenangemessenheit herstellen: Die Identifizierung von Forschungsthemen mit übergeordnetem Interesse für Akteure aus Wissenschaft und Praxis ist eine Kernvoraussetzung für eine fruchtbare Zusammenarbeit in einem Reallabor. Dabei kommt es auch immer wieder zu Interessenkonflikten. Abschnitt 3.1 zeigt, wie ein solcher Verständigungsprozess zwischen verschiedenen Akteuren ablaufen kann.

2) Räumliche Angemessenheit gestalten: In einem Reallabor werden, wie oben ausgeführt, vielfältige Ziele verfolgt. Ein Reallabor sollte einen abgegrenzten und zu seinen Zielen und Themen passenden Bezugsraum aufweisen, der mit den zu bearbeitenden Transformationsprozessen korrespondiert. Ein solcher klarer Bezugsraum macht ein Reallabor auch attraktiv für Akteure aus der Praxis. Die Frage nach diesem adäquaten Bezugsraum eines Reallabors wird in Abschnitt 3.2 diskutiert.

3) Zeitliche Angemessenheit herstellen: Neben der angemessenen räumlichen Gestaltung stellt auch die zeitliche Gestaltung transformativer Forschungsprozesse eine große Herausforderung für ein Reallabor dar (Abschnitt 3.3). Die Laufzeit eines Reallabors und die Dauer der transdisziplinären Projekte müssen den zu bearbeitenden Transformationsprozessen angemessen sein. Innerhalb dieser Zeitspannen gilt es jeweils, Passung zwischen dem Zeittakt der Forschung und anderen Abläufen, etwa in der Stadtplanung oder der Wirtschaft, herzustellen. Auch der Aufbau eines Reallabors als Infrastruktur, die Etablierung einer Kooperation zwischen Akteuren aus der Praxis und Wissen- 
schaft sowie die in einem Reallabor erforderlichen Reflexionsprozesse (s. unten) benötigen Zeit.

4) Angemessene Akteursrollen etablieren: Die Angemessenheit muss auch in der Zusammensetzung der beteiligten Akteure gesucht werden, ist diese doch untrennbar mit dem Anspruch der transdisziplinären Arbeitsweise in einem Reallabor verbunden. In welchen Rollen und in welchen Intensitäten eine Einbeziehung der verschiedenen Akteure aus Wissenschaft und Praxis im Reallabor angemessen sein kann, wird in Abschnitt 3.4 erläutert.

5) Experimentell-reflexive Arbeitsweise fördern: Will ein Reallabor lernfähig bleiben, muss es ein hohes Maß an Flexibilität und einen stark reflexiven Forschungsstil pflegen. Sowohl die Durchführung der transdisziplinären Projekte als auch die Passung zwischen diesen und deren Zusammenspiel mit den übergreifenden Aufgaben und der Infrastruktur auf Ebene des Reallabors bieten jeweils einen wichtigen Ansatzpunkt für die Reflexion. Aufgrund des transdisziplinären und transformativen Vorgehens im Reallabor erlangt zudem die Reflexion der eigenen Rolle der beteiligten Wissenschaftler(innen) große Bedeutung. Die experimentell-reflexive Arbeitsweise wird in Abschnitt 3.5 näher ausgeführt.

Die hier kurz skizzierten Designprinzipien werden im Folgenden detaillierter ausgeführt und mit Beispielen aus den BaWü-Labs verdeutlicht. Dabei ist zu beachten, dass ein Reallabor nach Ansicht der Autor(inn)en nicht als ein geschlossenes Labor in einen spezifischen Kontext eingepasst wird, sondern dass es einen Ausschnitt der Realität aufgreift und diesen zum Reallabor ausgestaltet.

\subsection{Problem- und Themenangemessenheit herstellen}

Ein Reallabor ermöglicht die exemplarische Bearbeitung relevanter transformationsbezogener Fragen, um übertragbare Ergebnisse für ähnliche Problemstellungen in anderen Kontexten zu liefern. Die übergeordnete Themenstellung wird dabei in der Regel bei Einrichtung eines Reallabors schon vorgezeichnet. Zur Bearbeitung dieser Themenstellung dienen insbesondere die transdisziplinären Projekte, deren jeweiliger thematischer Fokus zwischen Akteuren aus Wissenschaft und Praxis festgelegt wird. Dies erfordert einen gemeinsamen Verständigungsprozess aller beteiligten Akteure über Begriffe, Zielstellungen und Methoden. Das Reallabor Spacesharing - Nutzungsintensivierung von Bestandsgebäuden durch Mehrfachnutzung und dynamische Programmierung (BaWü-Lab Spacesharing) etablierte dazu, neben den Jour Fixes im Kernteam, regelmäßige Workshops mit seinen Kooperationspartnern und themenspezifisch mit den Nutzer(inne)n, um die Zielsetzungen, erreichte Zwischenergebnisse, Modifikationen und nächsten Schritte des Reallabors abzustimmen. Die Themenstellungen der 
Workshops bauten aufeinander auf, die gewählten Formate orientierten sich am Stand des Projektverlaufes. Die Methoden der Zusammenarbeit in diesen Workshops folgten etablierten Moderationsformen, diese wurden teilweise kombiniert und im Verlauf ad hoc geöffnet, um der Workshop-Dynamik zu folgen und dem optimalen Austausch der Partner Raum zu geben.

Die Auswahl der Themenstellung für ein transdisziplinäres Projekt wirkt sich auch auf den Kreis der potentiell beteiligten Akteure aus. Bei der Eingrenzung einer Problemstellung ist dementsprechend die Relevanz für unterschiedliche Akteure zu bedenken, wobei gleichermaßen Forschungs-, Praxis- und Bildungsziele berücksichtigt werden sollten. Dabei bietet es sich an, auch bei der Schärfung der Themen iterativ vorzugehen, da nach Di Giulio et al. (2016) der Nutzen für die Akteure nicht immer zu Beginn fassbar ist, was die frühe Eingrenzung der Problemstellung erschwert.

Da bei den verschiedenen Akteuren häufig unterschiedliche Erwartungen in Bezug auf die Ziele eines transdisziplinären Projekts bestehen, ist es wichtig, diese Erwartungen von Beginn der Zusammenarbeit an transparent zu machen - und auf ihre Realisierbarkeit hin zu überprüfen. Dabei gilt es immer auch zu bedenken, wann welches (Zwischen-)Ergebnis nötig ist, und zwar mit Blick auf die Forschungs-, Praxis- und Bildungsziele, auf Ebene des Reallabors und der transdisziplinären Projekte.

Bei der thematischen Ausgestaltung eines Reallabors als Ganzes ist es wichtig, sich bewusst zu machen, in welchem Kontext das Reallabor steht. Welche politischen, ökonomischen, gesellschaftlichen und kulturellen Prozesse laufen gerade ab? Befindet sich der bearbeitete ,Raum' in einer Phase des Aufbruchs, der Konsolidierung oder des Abschwungs - oder ist er stabil? So war für das Reallabor Wissensdialog Nordschwarzwald (BaWü-Lab WiNo) (s. a. Steckbrief im Anhang dieses Buchs) prägend, in der Phase der Identitätsfindung der Nationalparkregion tätig zu sein. Ein solcher Kontext eröffnet einem Reallabor auf der einen Seite Gestaltungsmöglichkeiten, z. B. bei der Mitentwicklung touristischer Leitbilder, auf der anderen Seite war es beim BaWü-Lab WiNo so, dass zentrale Akteure der Region noch in der Rollenfindung und mit der Verarbeitung der politischen Debatte um die Gründung des Nationalparks befasst waren, was den Aufbau der Zusammenarbeit und die gemeinsame Themenfindung erschwerte.

\subsection{Räumliche Angemessenheit gestalten}

Ein Reallabor benötigt einen angemessenen Bezugsraum, der je nach der inhaltlichen Ausrichtung eines Reallabors sehr unterschiedlich beschaffen sein kann. Denn ein Reallabor kann sich auf Städte bzw. Stadtbezirke, ländliche Regionen oder Schutzgebiete (wie z. B. Naturparks), auf Gebäudekomplexe oder auf Insti- 
tutionen beziehen oder auch auf virtuelle Räume (wie z. B. die vernetzten Orte einer Wertschöpfungskette) (Schäpke et al. 2017). Wesentlich für die Arbeitsund Kommunikationsfähigkeit eines Reallabors ist dabei, dass dessen Bezugsraum von den im Reallabor mitwirkenden Akteuren auch anerkannt und geteilt wird (Bachinger und Rhodius 2017).

Der Abgrenzung eines Reallaborraums können ganz verschiedene Kriterien zugrunde liegen (Parodi et al. 2018): So bezog sich das Reallabor für nachhaltige Mobilitätskultur (BaWü-Lab RNM) auf den Pendlerverkehr Stuttgarts und damit auf einen funktional abgegrenzten Raum (s. a. Steckbrief im Anhang dieses Buchs). Demgegenüber arbeitete das BaWü-Lab R131 in einem Stadtquartier und damit einem administrativ festgelegten Gebiet. Mit der engen Anlehnung an den Nationalpark Schwarzwald orientierte sich auch das BaWü-Lab WiNo an einem administrativen Raum, zugleich verwies dieser aber auch auf den mehr oder weniger homogenen Naturraum des Nordschwarzwalds. Das Reallabor STADTRAUM-BILDUNG - Reallabor für die nachhaltige Planung von Bildungslandschaften und die Integration von Aus- und Umbauten von Schulgebäuden (BaWü$L a b S R B$ ) hingegen arbeitete in verschiedenen Schulen und deren angrenzenden Stadträumen, das BaWü-Lab EnSign - Reallabor für einen klimaneutralen Innenstadtcampus am Campus der Universität Stuttgart.

Die Abgrenzung des Bezugsraums aufgrund funktionaler, naturräumlicher oder administrativer Kriterien stellt jedoch nur eine Komponente dar. Wichtig für die Akzeptanz des Bezugsraums durch die beteiligten Akteure aus der Praxis ist es auch, dass es gelingt, an individuelle Raumerfahrungen anzuknüpfen und sich damit auf Räume zu beziehen, die auch ausreichend Identifikation bieten (Weichhart 2008). So wurde der Bezug auf den Naturraum Nordschwarzwald von den Wissenschaftler(inne)n des BaWü-Labs WiNo als sehr prägend für die Zusammenarbeit mit Akteuren empfunden, da diese in ihrer Sozialisierung tatsächlich vom Leben in diesem Naturraum geprägt waren. Im $B a W u ̈-L a b S R B$ hingegen wird der Stadtraum als ein offenes Element begriffen, in dem die Schule als das zentrale Element für die Wissensgesellschaft von heute steht - als Lern- und Wissensraum. Das BaWü-Lab Spacesharing untersuchte untergenutzte Räume in der Stadt, kartierte und kategorisierte diese, mit der Zielsetzung, eine digitale Angebots- und Buchungs-Plattform aufzubauen, die diese Räume öffnet, überregional vermittelt und wiederum für Transformationsprozesse zugänglich macht.

Dort, wo sich ein gemeinsamer Raumbezug z. B. aufgrund einer sehr großen oder heterogenen Bezugsregion nicht herstellen lässt, kann eine sogenannte virtuelle Region im Sinne der Kommunikation über gemeinsame Problemstellungen entstehen (Bachinger und Rhodius 2017; Herrschel 2009). Ein Beispiel hierfür stellt 
das Städtenetzwerk „100 resilient cities“ ${ }^{\text {(14 }}$ dar, in dem sich Großstädte bei der Erarbeitung von Resilienzstrategien zur Bewältigung zukünftiger Herausforderungen unterstützen. Denkbar ist auch, dass sich die verschiedenen transdisziplinären Projekte auf unterschiedliche Teile des Bezugsraums eines Reallabors beziehen (Parodi et al. 2018): Beispielsweise konzentrierten sich einzelne Realexperimente im BaWü-Lab RNM auf die Innenstadt, nicht die gesamte Pendlerregion. Im BaWü-Lab WiNo kristallisierten sich je nach Thema der neun Teilprojekte unterschiedliche Bezugsräume heraus, die von klein und punktuell auf ein Tal im Nationalpark begrenzten Räumen (z. B. beim Thema waldbauliche Experimente) bis hin zum weiteren ländlichen Umland des Nationalparks (z. B. beim Thema Mobilität) und einzelnen in größerer Ferne umgebenden Großstädten (beim Thema Tourismus) reichten.

Die Bezugsräume der transdisziplinären Projekte stehen also nicht zwangsläufig schon mit der Initiierung eines Reallabors und unverändert über den gesamten Zeitraum fest. Die jeweils angemessenen Bezugsräume müssen im Austausch zwischen den Akteuren definiert und im Verlauf überprüft werden (s. auch Waitz et al. 2018), auf Ebene eines transdisziplinären Projekts und gegebenenfalls eines Reallabors als Ganzem. Dabei ist darauf zu achten, dass nicht einzelne Akteure aus Wissenschaft oder Praxis die alleinige Deutungshoheit gewinnen, sondern der Raumbezug gleichberechtigt festgelegt wird (Bachinger und Rhodius 2017).

Ist der Bezugsraum eines Reallabors festgelegt, ist es für die Identifikation der beteiligten Akteure mit dem Reallabor hilfreich, wenn dieses sichtbar und zugänglich wird, wie z. B. durch das Quartiersbüro „Zukunftsraum“ im BaWü-Lab R131.

\subsection{Zeitliche Angemessenheiten herstellen}

Die Frage einer angemessenen zeitlichen Planung ist für die Zusammenarbeit von Akteuren aus Wissenschaft und Praxis von großer Bedeutung und damit Thema eines jeden transdisziplinären Projektes (Defila et al. 2006). Dabei kommen in der Regel die Frage unterschiedlicher Zeitrhythmen der beteiligten Akteure, die Notwendigkeit eines flexiblen Zeitrahmens und der Bedarf nach einer ausreichend langen Projektzeitspanne zur Sprache - Aspekte, die sich auch in einem Reallabor wiederfinden.

- Unterschiedliche Zeitrhythmen: Zu Beginn eines Projektes steht an, dass sich die beteiligten Akteure wechselseitig mit den Arbeitszyklen der anderen vertraut machen. So gibt es beispielsweise Berufszweige, die stark saisonal arbeiten (z. B. Landwirtschaft, Tourismus), was z. B. bei der Planung von Work-

14 http://www.100resilientcities.org (zugegriffen am 04.02.2018). 
shops einkalkuliert werden sollte. Zeitdruck kann beispielsweise entstehen, wenn Praxisentscheidungen zu einem Zeitpunkt nötig sind, zu dem noch keine Forschungsergebnisse vorliegen. Bezogen auf den Transformationsprozess können auch die Zeitrhythmen von Akteuren ausschlaggebend sein, die nicht am Reallabor beteiligt sind (Bachinger und Rhodius 2017). Dies war beispielsweise im BaWü-Lab WiNo der Fall, als Wissenschaftler(innen) auf die Betretungsgenehmigung für die Einrichtung ihres Realexperiments warteten, der Nationalpark jedoch gerade erst an der Einteilung des Parkgebietes in verschiedene Schutzzonen arbeitete und deshalb noch internen Abstimmungsbedarf hatte.

- Flexibler Zeitrahmen: Die experimentelle Arbeitsweise und die Arbeit an Transformationsprozessen, deren Dynamik weit über die Grenzen des Reallabors hinausgehen kann, macht den Umgang mit unvorhersehbaren Entwicklungen besonders nötig. Hierfür bedarf es ausreichender Pufferzeiten und flexibler Zeitkorridore.

- Ausreichende Zeitdauer zur Vertrauensbildung: Ein Reallabor arbeitet in der Regel mit mehr oder weniger stark ausgebildeten Akteursnetzwerken, nicht innerhalb streng hierarchischer Strukturen (Hollstein et al. 2017). Es ist daher darauf angewiesen, in diesen Netzwerken Vertrauen, gemeinsame Wertorientierungen und damit tragfähige Arbeitsbeziehungen aufzubauen - und das erfordert Zeit. Auch Wissen entsteht über längere Zeiträume und auf der Basis stabiler Beziehungen zu Akteuren aus der Praxis (Foss 1999).

Im Vergleich zu isoliert ablaufenden Forschungsprojekten bedarf es im Fall transdisziplinärer Projekte im Rahmen eines Reallabors einer zeitlichen Planung, die sich in noch stärkerem Maß weg von der Logik der Forschung hin zur Logik der Praxis bewegt. Dies deshalb, weil Reallabore darauf abzielen, Transformationsprozesse in der Lebenswelt zu initiieren, durchzuführen und zu begleiten. Solche Prozesse ${ }^{15}$ bedürfen oft deutlich längerer Zeithorizonte als den Horizont eines klassischen, zumeist ein- bis dreijährigen Forschungsprojekts. Die Zeitplanung eines Reallabors und der darin stattfindenden transdisziplinären Projekte muss sich an die angestrebten Praxisziele und die dafür notwendigen Aktivitäten anpassen, um gesellschaftlich relevant zu bleiben.

15 Z. B. Dynamik in Ökosystemen, Infrastrukturtransformationen oder legislative Entscheidungen. 


\subsection{Angemessene Akteursrollen etablieren}

In einem Reallabor kooperieren Akteure aus der Wissenschaft und aus der Praxis. Dies geschieht zumindest auf zwei Ebenen, auf der des Reallabors als Ganzes und auf Ebene eines einzelnen transdisziplinären Projektes. ${ }^{16}$ Auf diesen Ebenen können dieselben Akteure in unterschiedlichen Rollen beteiligt sein. Über die Laufzeit eines Reallabors kann sich die Rolle von Akteuren auch verändern. Auf der Ebene des Reallabors kommt Akteuren, die auch langfristig im Reallabor engagiert sind (z. B. Mitgliedern von Verwaltung, großen zivilgesellschaftlichen Gruppen, festangestellten Wissenschaftler(inne)n), tendenziell eher eine tragende Rolle zu. Dagegen können einzelne transdisziplinäre Projekte leichter eine niedrige Einstiegsschwelle für neue und nicht institutionell organisierte Akteure bieten, sich nur punktuell am Reallabor zu beteiligen (s. auch Seebacher et al. 2018a).

Die Rollen der beteiligten Akteure aus der Wissenschaft umfassen auf jeden Fall: $\mathrm{Zu}$ forschen, Prozesse anzustoßen und zu moderieren (,Facilitator'), Expertise einzubringen und Ergebnisse zu analysieren und zu reflektieren. Daneben können die Akteure aus der Wissenschaft aber auch zahlreiche andere Rollen innehaben, etwa: Für eine Idee zu werben, das Reallabor öffentlich zu vertreten oder das eigene Handeln als Teil des Transformationsprozesses zu reflektieren. Die ansonsten dominante Orientierung der Akteure aus der Wissenschaft an Forschungszielen (und sekundär an Bildungszielen bei Studierenden) wird in einem Reallabor um Praxisziele deutlich erweitert. Studierende stellen für ein Reallabor eine interessante Gruppe von Akteuren aus der Wissenschaft dar, da sie zwar Mitglieder des Wissenschaftssystems sind, aber oft noch nicht eingefahren sind in klassische wissenschaftsorientierte Rollen.

Akteure aus der Praxis sind in jedem Fall in der Rolle gefragt, lebensweltliche Problemlagen zu identifizieren, die eine Bearbeitung im Reallabor erfahren sollen, als Träger von unverzichtbarem Praxiswissen (z. B. implizites oder lokales Wissen), als Multiplikatoren und gegebenenfalls als langfristige Träger der Ergebnisse, nachdem in einem Reallabor die Bearbeitung eines Themas abgeschlossen wurde. Je nach Problemstellung kommt ihnen dabei auch die Rolle zu, die Perspektiven bestimmter betroffener oder ausgeschlossener Gruppen oder unterrepräsentierte Anliegen einzubringen, ohne dass dadurch jedoch eine demokratische Legitimation erreicht werden könnte (s. auch Alcántara et al. 2018; Eckart et al. 2018). Im Forschungsprozess können Akteure aus der Praxis auch als Co-Forschende einbezogen sein, um transdisziplinäre Projekte mitzuplanen (Co-Design) und durchzuführen sowie Ergebnisse auszuwerten und zu integrieren. So entstan-

16 Darüber hinaus ist eine Beteiligung auf Ebene einzelner Methoden denkbar, wenn beispielsweise ein transdisziplinäres Projekt mit einer intensiven partizipativen Themenfindungsphase beginnt. 
den etwa die Forschungsschwerpunkte des BaWü-Labs R131 aus den Wünschen des BürgerForums „Nachhaltige Oststadt - Zukunft aus Bürgerhand“. Im BaWü$L a b$ RNM entstand die Themensetzung des Reallabors aus den Problemlagen in der Stadt Stuttgart, die durch motorisierten Individualverkehr induziert wurden, und der daraus resultierenden öffentlichen Debatte, die von der Universität aufgegriffen wurde. Die Themenschwerpunkte der transdisziplinären Projekte wurden dann gleichberechtigt von Akteuren aus der Praxis und Akteuren aus der Wissenschaft eingebracht. Im Reallabor Nachhaltige Transformation der Textilwirtschaft am Standort Dietenheim (BaWü-Lab Dietenheim zieht an) arbeitete von Beginn an die Stadtverwaltung mit der Universität Ulm und Unternehmern aus der Textilindustrie zusammen.

Die in einem Reallabor zu leistende Annäherung der Rollen von Akteuren aus der Wissenschaft und aus der Praxis ist nicht immer einfach (s. auch Seebacher et al. 2018b). Eingespielte Rollen zu verlassen, kostet Überwindung (und muss nicht notwendigerweise zu einem besseren Ergebnis führen), unerwartete Rollenanforderungen können belastend oder kränkend wirken, Erwartungen können enttäuscht werden. Selbst wenn die Rollenverschiebungen gut gelingen, kosten sie Zeit und bedeuten unter Umständen anstrengende Aushandlungsprozesse. Dabei stellt die Grenzziehung zwischen bezahlter Arbeit (z. B. von Wissenschaftler(inn)en, Mitarbeiter(inne)n von Unternehmen und Verwaltungsangestellten) gegenüber zivilgesellschaftlichem Ehrenamt und der Arbeit von Studierenden eine Sollbruchstelle in der Kooperation dar, mit der vorsichtig umgegangen werden muss. ${ }^{17}$ Auch die Rollen, die sich aus dem Aufbau und der Pflege der Infrastruktur ergeben, bergen Konfliktpotenzial.

Bei allen Tücken der Rollenfindung und -aushandlung in einem Reallabor gilt es, nicht zu vergessen, dass in einem Reallabor auch gänzlich neue Rollen ermöglicht werden, die Engagement, wissenschaftliche Haltung und Lernbereitschaft anders als üblich kombinieren. Diese Rollen bieten insbesondere Anknüpfungspunkte für diejenigen, die sich bereits mit einem Erkenntnisinteresse in Transformationsprozessen engagieren. Studentische Initiativen im Kontext Nachhaltiger Entwicklung können hier idealtypisch als Beispiele genannt werden, aber auch individuelle Akteure, die als ,Change Agents ${ }^{6}$ Transformationsprozesse in nichtstandardisierten Rollen vorantreiben.

Die Intensität der Beteiligung von Akteuren aus der Praxis in einem Reallabor kann gestaffelt sein, so dass z. B. Aktivitäten zur Information und Diskussion eine Vorstufe für intensivere Beteiligung darstellen (s. Eckart et al. 2018). Die In-

17 Ein Weg, hier einen Ausgleich zu schaffen, sind Formen der Aufwandsentschädigung wie die Ehrenamtspauschale, Lehraufträge, Übernahme von Reisekosten, oder die teilweise Anrechnung von zivilgesellschaftlichem Engagement als Teil der Dienstaufgaben auf Seiten der Akteure aus der Wissenschaft oder der Verwaltung. 
tensivierung der Beteiligung geht oft mit einer Verschiebung der Rollen einher, sowohl auf Ebene der einzelnen transdisziplinären Projekte als auch auf Ebene des Reallabors.

\subsection{Experimentell-reflexive Arbeitsweise fördern}

Ein Reallabor bietet sowohl einen Rahmen für experimentelle Vorgehensweisen (insbesondere Realexperimente) zur Erprobung und Analyse von Transformationsprozessen als auch zur systematischen Reflexion der transdisziplinären Prozesse, der Zusammenarbeit und der Ergebnisse. Eine solche experimentellreflexive Arbeitsweise hat vielfältige Vorteile. Während durch experimentelle Arbeitsschritte Erfahrungen gesammelt, Daten erzeugt und praktische Wirkungen angestoßen werden, dienen reflexive Arbeitsschritte der Überprüfung und Integration des gewonnenen Wissens, aber auch dem Überdenken der eigenen Rolle und dem Hinterfragen eigener grundlegender Überzeugungen der beteiligten Akteure aus Wissenschaft und Praxis.

Wichtig ist dabei zu verstehen, dass die in einem solchen Zyklus erarbeiteten Einsichten nicht nur in die wissenschaftlichen und praktischen Diskurse zurückgespielt oder real umgesetzt werden, sondern auch das Reallabor selbst verändern. Ein Reallabor entwickelt sich in seiner experimentell-reflexiven Arbeitsweise:

- Die thematische, räumliche, zeitliche und akteursbezogene Angemessenheit können immer wieder überprüft und angepasst werden.

- Der Zielhorizont aus Forschungs-, Praxis- und Bildungszielen kann aktualisiert, Zielkonflikte können bearbeitet werden.

- Wissensbestände können integriert und für folgende transdisziplinäre Projekte im Reallabor aufgegriffen werden.

- Erfahrungen aus gescheiterten oder unerwartet verlaufenen transdisziplinären Projekten können aufgegriffen werden.

- In den transdisziplinären Projekten können Methoden adaptiert, entwickelt und getestet werden.

- Bei den Beteiligten können Kompetenzen in der transdisziplinären Zusammenarbeit aufgebaut werden.

Eine experimentell-reflexive Arbeitsweise im Sinne eines emanzipatorischen Lernens (Schneidewind und Singer-Brodowski 2015) kann die beteiligten Akteure darin unterstützen, eigene Problemlösungsideen zu entwickeln und zu erproben (Parodi et al. 2016b). Wenn die Akteure die Wirksamkeit der von ihnen erprobten 
Lösungsansätze gemeinsam reflektieren und bewerten, kann ein Lernkreislauf in Gang gesetzt werden. Dieser bewirkt idealerweise, dass das Wissen auch eine Berücksichtigung im Handeln in anderen Kontexten erfahren kann (Bachinger und Rhodius 2018). Durch den Mechanismus, die Erfahrungen eines transdisziplinären Projektes für Folgeprojekte fruchtbar zu machen, wird ein Reallabor zu einer lernenden Organisation (s. z. B. Barth 2015). Als solche bietet es gleichermaßen Flexibilität gegenüber veränderten Rahmenbedingungen und das Potential zur internen Weiterentwicklung. Je nach der Tiefe des Engagements der Beteiligten kann die Reflexion auch einen anstrengenden Schritt darstellen, der das eigene Selbstbild und professionelle Rollenverständnis betrifft.

Es ist davon auszugehen, dass dem Idealbild eines sich durch Reflexion fortlaufend entwickelnden Reallabors Hemmnisse entgegenstehen. So bringen Akteure aus der Praxis (z. B. aufgrund ihrer Rolle außerhalb des Reallabors oder ihrer institutionellen Verankerungen) verschiedene Sichtweisen ein, die sich nicht in jedem Fall zusammenführen lassen. Auch werden in der Regel nicht alle Sichtweisen und Wissensbestände in gleichem Umfang in eine Reflexion integriert (Mayntz 2009). Umso wichtiger ist es, dass Zeitfenster und Organisationsstrukturen für eine gemeinsame Reflexion in einem Reallabor verankert werden. So wurde im BaWü-Lab WiNo eine Querschnitts-Arbeitsgruppe gegründet, in der jedes Teilprojekt vertreten war. Etwa vierteljährlich traf sich diese Gruppe, um sich über den Arbeitsstand und Fragen der Qualitätssicherung transdisziplinären Arbeitens auszutauschen. Die experimentell-reflexive Arbeitsweise macht aber auch eine gewisse Flexibilität überhaupt erst möglich. So zeigte sich im BaWü-Lab R131 in der ersten Runde von Realexperimenten etwa, dass die Partizipation der Bevölkerung entgegen der Absicht zu schwach realisiert wurde. Basierend auf dieser Einsicht des Reallaborteams wurde diese Schieflage in der Konstellation der Akteure in der zweiten Runde mit der Realexperimentreihe „Dein NachhaltigkeitsExperiment" (s. Trenks et al. 2018) korrigiert. Im BaWü-Lab Dietenheim zieht an zeigte sich im Projektverlauf, dass eine Wiederbelebung der Innenstadt durch nachhaltige Modegeschäfte kaum Aussicht auf Erfolg haben würde, so dass stattdessen ein Messeformat entwickelt wurde. Für die BaWü-Labs stellten die Workshops und Impulse der Begleitforschung wiederkehrende Anlässe zur Reflexion der eigenen Arbeitsweise dar.

Zur gemeinsamen, öffentlichen Reflexion ihrer Arbeit hatten BaWü-Labs beider Förderlinien die Reallabor Lounge initiiert. Diese verband den gesellschaftlichen Diskurs zur Nachhaltigkeitsstrategie Baden-Württemberg mit Erfahrungen aus den Reallaboren und gab der Öffentlichkeit Raum, ihre Fragen mit Expert(inn)en zu diskutieren. Daraus resultierende Wissensgewinne integrierten die BaWü-Labs in ihre weitere Arbeit. 


\section{Ziele und Designprinzipien in einem Reallabor: Lessons Learnt}

Reallabore stellen einen Rahmen dar, Forschungs-, Praxis- und Bildungsziele synergetisch zu verfolgen. Bei der Konzeption eines Reallabors gilt es, die oben angeführten Designprinzipien zu beachten, um die Ziele optimal verfolgen zu können. Dabei lassen sich die Designprinzipien keineswegs 1:1 den Zielen zuordnen, sondern liegen quer zu ihnen: Räumliche Angemessenheit beispielsweise bezieht sich auf die Auswahl bearbeitbarer Untersuchungsräume, die von einem Transformationsprozess betroffenen Räume sowie die Gestaltung einer geeigneten Lernumgebung. Tabelle 1 bildet ein Schema aus Zieldimensionen und Designprinzipien. Darin werden beispielhafte Lessons Learnt aus den BaWü-Labs aufgeführt.

Tabelle 1: Wie lassen sich die Ziele eines Reallabors mithilfe der Designprinzipien erreichen? Beispielhafte Lessons Learnt aus den BaWü-Labs

\begin{tabular}{|c|c|c|c|}
\hline $\begin{array}{l}\text { Design- } \\
\text { prinzipien }\end{array}$ & Forschungsziele & Praxisziele & Bildungsziele \\
\hline 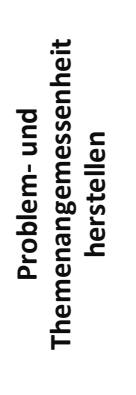 & $\begin{array}{l}\text { Exemplarisch } \\
\text { Fragen bearbei- } \\
\text { ten, die über- } \\
\text { tragbare Ergeb- } \\
\text { nisse liefern }\end{array}$ & 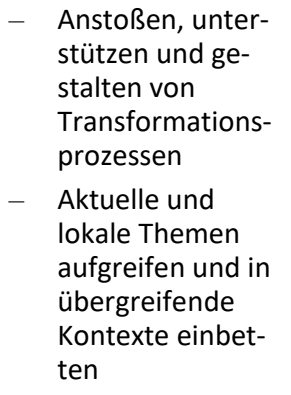 & $\begin{array}{l}\text { Empowerment } \\
\text { von Akteuren } \\
\text { im Hinblick auf } \\
\text { zukünftige } \\
\text { Transformations- } \\
\text { prozesse }\end{array}$ \\
\hline 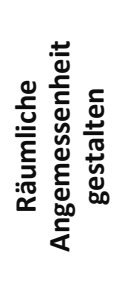 & 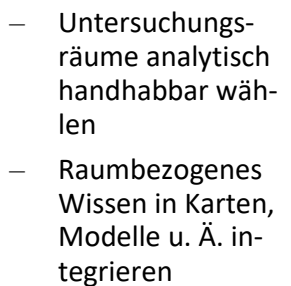 & $\begin{array}{l}\text { - Räumliche Skalen } \\
\text { (z. B. Quartier, } \\
\text { Einzugsgebiet...) } \\
\text { identifizieren, um } \\
\text { Themen lokal be- } \\
\text { arbeitbar zu ma- } \\
\text { chen }\end{array}$ & $\begin{array}{ll}- & \text { Reallabor als } \\
\text { „Lernumgebung“ } \\
\text { gestalten } \\
\text { - } & \text { Identifizierung } \\
\text { der Akteure mit } \\
\text { dem Raum er- } \\
\text { möglichen und } \\
\text { aufgreifen }\end{array}$ \\
\hline
\end{tabular}




\begin{tabular}{|c|c|c|c|}
\hline $\begin{array}{l}\text { Design- } \\
\text { prinzipien }\end{array}$ & Forschungsziele & Praxisziele & Bildungsziele \\
\hline 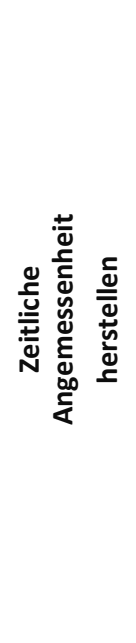 & 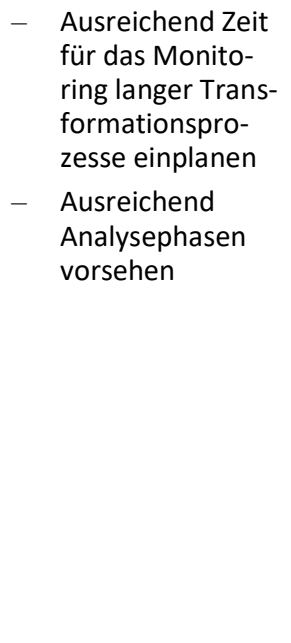 & $\begin{array}{l}\text { - An die Dauer und } \\
\text { Taktung realer } \\
\text { Transformations- } \\
\text { prozesse an- } \\
\text { schließen } \\
\text { - } \\
\text { Strukturen auf- } \\
\text { bauen, die eine } \\
\text { langfristige Be- } \\
\text { arbeitung von } \\
\text { Themen (auch } \\
\text { außerhalb des } \\
\text { Reallabors) er- } \\
\text { möglichen } \\
\text { Flexiblen Zeit- } \\
\text { rahmen schaffen, } \\
\text { um mit unerwar- } \\
\text { teten Ereignissen } \\
\text { umzugehen }\end{array}$ & $\begin{array}{ll}- & \text { Reflexionsphasen } \\
& \text { vorsehen } \\
- & \text { Zyklische Arbeits- } \\
& \text { weisen etablieren } \\
-\quad & \text { Zeit für individu- } \\
& \text { elle Bildungspro- } \\
\text { zesse und für } & \text { Veränderungs- } \\
\text { prozesse als ler- } & \text { nende Organisa- } \\
\text { tion einplanen }\end{array}$ \\
\hline 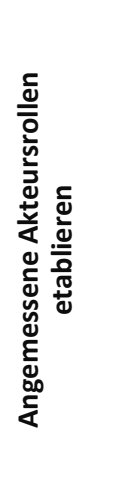 & 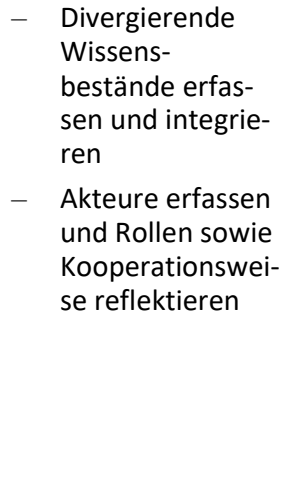 & 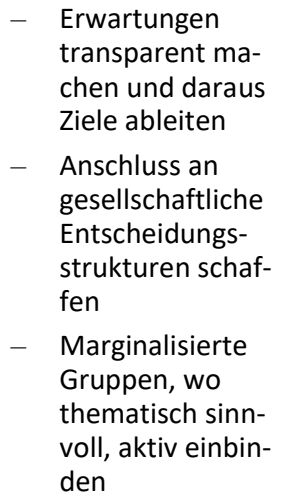 & $\begin{array}{l}\text { Gegenseitiges } \\
\text { Lernen („mutual } \\
\text { learning“) ver- } \\
\text { schiedener Ak- } \\
\text { teure von- und } \\
\text { miteinander för- } \\
\text { dern } \\
\text { Rollenklärung als } \\
\text { Gelegenheit zur } \\
\text { Selbstreflexion } \\
\text { nutzen }\end{array}$ \\
\hline 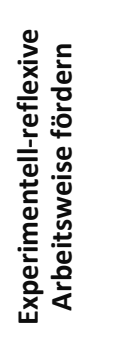 & $\begin{array}{l}\text { - } \text { Reallabordesign } \\
\text { so auslegen, dass } \\
\text { Rückschläge und } \\
\text { Scheitern als Teil } \\
\text { der Arbeitsweise } \\
\text { genutzt werden } \\
\text { - } \\
\text { Selbstreflexion } \\
\text { auch als For- } \\
\text { schungsaufgabe } \\
\text { auffassen }\end{array}$ & $\begin{array}{l}\text { Nicht-intendierte } \\
\text { Folgen und Reak- } \\
\text { tionen innerhalb } \\
\text { und außerhalb } \\
\text { des Reallabors } \\
\text { erfassen }\end{array}$ & $\begin{array}{l}\text { Erfahrungstrans- } \\
\text { fer zwischen un- } \\
\text { terschiedlichen } \\
\text { transdisziplinären } \\
\text { Projekten ermög- } \\
\text { lichen } \\
\text { Evaluation als } \\
\text { Gelegenheit zum } \\
\text { Lernen nutzen }\end{array}$ \\
\hline
\end{tabular}


Zur Gestaltung eines Reallabors mit eigenem Profil empfehlen die Autor(inn)en, die Tabelle zu nutzen und für jede Zelle bereits in der Planungsphase eigene Designentscheidungen zu dokumentieren - im Wissen darum, dass sich diese im Verlauf der Reallaborarbeit noch deutlich wandeln werden. Die einzelnen Lessons Learnt in der Tabelle sollen hierbei als Inspiration dienen, je nach Fokus eines Reallabors kann hier aber auch ganz anderen Punkten Priorität zukommen.

Auch für den Evaluations-, Reflexions- und Veränderungsprozess eines Reallabors kann die vorliegende Tabelle als Reflexionsschema dienen. Die Autor(inn)en schlagen vor, die (leere) Tabelle zu nutzen, um divergierende Perspektiven unterschiedlicher, am Reallabor beteiligter Akteure zu erheben und darauf aufbauend das spezifische Design des Reallabors Schritt für Schritt abzustimmen. Schließlich kann anhand des Schemas für einzelne transdisziplinäre Projekte oder einzelne Methoden überprüft werden, inwieweit sie in das Reallabor passen bzw. ob sie dessen Potential in vollem Umfang nutzen.

\section{Dank}

Dieser Text wäre ohne den vertrauensvollen und kooperativen Austausch der BaWü-Lab-Teams untereinander nicht möglich gewesen; stellvertretend möchten wir Annika Arnold, Felix M. Piontek und Ines-Ulrike Rudolph für die Bereitstellung von Beispielen aus ihren jeweiligen Reallaboren danken. Die Autorinnen und Autoren danken auch Elke Häußler, Niko Schäpke, Andreas Seebacher und Christina West für die Diskussionen über frühere Fassungen des Textes im Rahmen des internen Reviews. Insbesondere möchten sie den zwei anonymen externen Gutachter(inne)n für die konkreten Hinweise zur Verbesserung des Textes danken. Schließlich danken sie den beiden Herausgebenden, Rico Defila und Antonietta Di Giulio, für ihre detaillierten und zielführenden Rückmeldungen zum Text.

\section{Literatur}

Alcántara, S., Quint, A., \& Seebacher, A. (2018). Der Partizipationsmythos „Partizipation in Reallaboren muss repräsentativ sein“. In R. Defila \& A. Di Giulio (Hrsg.), Transdisziplinär und transformativ forschen. Eine Methodensammlung (S. 137-141). Wiesbaden: Springer VS.

Arnold, A., \& Piontek, F. M. (2018). Zentrale Begriffe im Kontext der Reallaborforschung. In R. Defila \& A. Di Giulio (Hrsg.), Transdisziplinär und transformativ forschen. Eine Methodensammlung (S. 143-154). Wiesbaden: Springer VS. 
Arnstein, S. (1969). A Ladder Of Citizen Participation. Journal of the American Planning Association, 35 (4), (S. 216-224).

Bachinger, M., \& Rhodius, R. (2018). Anforderungen an die Governance von transdisziplinären Lernprozessen in Reallaboren. Das Beispiel des „Wissensdialogs Nordschwarzwald (WiNo)“. Berichte. Geographie und Landeskunde, 91 (1), (S. 81-96). (im Druck).

Barth, M. (2015). Implementing Sustainability in Higher Education: Learning in an Age of Transformation. Routledge studies in sustainable development. London: Routledge.

Beecroft, R., \& Dusseldorp, M. (2009). TA als Bildung. Ansatzpunkte für Methodologie und Lehre. Technikfolgenabschätzung - Theorie und Praxis, 18 (3), (S. 55-64).

Beecroft, R., \& Dusseldorp, M. (2012). Technikfolgen abschätzen lehren - Einleitung. In M. Dusseldorp, R. Beecroft (Hrsg.), Technikfolgen abschätzen lehren. Bildungspotenziale transdisziplinärer Methoden. Wiesbaden: Springer VS.

Beecroft, R., \& Parodi, O. (2016). Reallabore als Orte der Nachhaltigkeitsforschung und Transformation. Einführung in den Schwerpunkt. Technikfolgenabschätzung - Theorie und Praxis, 25 (3), (S. 4-8).

Bergmann, M., Jahn, T., Knobloch, T., Krohn, W., Pohl, C., \& Schramm, E. (2010). Methoden transdisziplinärer Forschung: Ein Überblick mit Anwendungsbeispielen. Frankfurt a. M., New York: Campus.

CASS \& ProClim- (1997). Forschung zu Nachhaltigkeit und Globalem Wandel - Wissenschaftspolitische Visionen der Schweizer Forschenden. Bern: ProClim-/SANW. https:// naturwissenschaften.ch/service/publications/75640-visionen-der-forschenden. Zugegriffen am 21.01.2018.

Defila, R., Di Giulio, A., \& Scheuermann, M. (2006). Forschungsverbundmanagement: Handbuch für die Gestaltung inter- und transdiziplinärer Projekte. Zürich: vdf Hochschulverlag.

Defila, R., \& Di Giulio, A. (2018). Reallabore als Quelle für die Methodik transdisziplinären und transformativen Forschens - eine Einführung. In R. Defila \& A. Di Giulio (Hrsg.), Transdisziplinär und transformativ forschen. Eine Methodensammlung (S. 935). Wiesbaden: Springer VS.

Di Giulio, A., Defila, R., \& Brückmann, Th. (2016). „Das ist halt das eine... Praxis, das andere ist Theorie" - Prinzipien transdisziplinärer Zusammenarbeit im Forschungsalltag. In R. Defila \& A. Di Giulio (Hrsg.), Transdisziplinär forschen - zwischen Ideal und gelebter Praxis. Hotspots, Geschichten, Wirkungen (S. 189-286). Frankfurt a. M., New York: Campus.

Dusseldorp, M. (2017). Zielkonflikte der Nachhaltigkeit. Wiesbaden: Springer Fachmedien.

Eckart, J., Ley, A., Häußler, E., \& Erl, Th. (2018). Leitfragen für die Gestaltung von Partizipationsprozessen in Reallaboren. In R. Defila \& A. Di Giulio (Hrsg.), Transdisziplinär und transformativ forschen. Eine Methodensammlung (S. 105-135). Wiesbaden: Springer VS.

Finke, P., \& Laszlo, E. (2014). Citizen Science: Das unterschätzte Wissen der Laien. München: Oekom. 
Foss, N. J. (1999). Networks, Capabilities and Competitive Advantage. Scandinavian Journal of Management, 15 (1), (S. 1-15).

Groß, M., Hoffmann-Riem, H., \& Krohn, W. (2005). Realexperimente. Ökologische Gestaltungsprozesse in der Wissensgesellschaft. Bielefeld: Transcript.

Grunwald, A. (2016). Nachhaltigkeit verstehen. München: Oekom.

Herrschel, T. (2009). Regionalisation, „virtual“ spaces and „real“ territories. A view from Europe and North America. International Journal of Public Sector Management, 22 (3), (S. 272-285).

Hollstein, B., Matiaske, W., \& Schnapp, K. (2017). Networked Governance: Taking networks seriously. In B. Hollstein, W. Matiaske \& K. Schnapp (Hrsg.), Networked Governance (S. 1-11). Berlin: Springer.

Krainer, L., \& Trattning, R. (Hrsg.) (2017). Kulturelle Nachhaltigkeit: Konzepte, Perspektiven, Positionen. München: Oekom.

Marquardt, E., \& West, C. (2016). Co-Produktion von Wissen in der Stadt. Reallabor „Urban Office - Nachhaltige Stadtentwicklung in der Wissensgesellschaft“" an der Universität Heidelberg. Technikfolgenabschätzung - Theorie und Praxis, 25 (3), (S. 26-31).

Mayntz, R. (2009). Über Governance. Institutionen und Prozesse politischer Regelung. Schriften aus dem Max-Planck-Institut für Gesellschaftsforschung, Bd. 62, Frankfurt a. M.: Campus.

Meyer-Soylu, S., Parodi, O., Trenks, H., \& Seebacher, A. (2016). Das Reallabor als Partizipationskontinuum - Erfahrungen aus dem Quartier Zukunft und Reallabor 131 in Karlsruhe. Technikfolgenabschätzung - Theorie und Praxis, 25 (3), (S. 31-40).

MWK (Ministerium für Wissenschaft, Forschung und Kunst Baden-Württemberg) (Hrsg.) (2013). Wissenschaft für Nachhaltigkeit. Herausforderung und Chance für das badenwürttembergische Wissenschaftssystem. Stuttgart. https://www.baden-wuerttemberg. de/fileadmin/redaktion/dateien/PDF/Broschüre_Wissenschaft_für_Nachhaltigkeit.pdf. Zugegriffen am 27.11.2017.

Parodi, O. (2015). The missing aspect of culture in sustainability concepts. In J. C. Enders \& M. Remig (Hrsg.), Theories of sustainable development (S. 169-187). London, New York: Routledge.

Parodi, O., Albiez, M., Beecroft, R., Meyer-Soylu, S., Quint, A., Seebacher, A., Trenks, H., \& Waitz, C. (2016a). Das Konzept „Reallabor" schärfen. Ein Zwischenruf des Reallabor 131: KIT findet Stadt. GAIA, 25 (4), (S. 284-285). doi: 10.14512/gaia.25.4.11.

Parodi, O., Beecroft, R., Albiez, M., Quint, A., Seebacher, A., Tamm, K., \& Waitz, C. (2016b). Von „Aktionsforschung“ bis „Zielkonflikte“ - Schlüsselbegriffe der Reallaborforschung. Technikfolgenabschätzung - Theorie und Praxis, 25 (3), (S. 9-18).

Parodi, O., Waitz, C., Bachinger, M., Kuhn, R., Meyer-Soylu, S., Alcántara, S., \& Rhodius R. (2018). Insights into and recommendations from three real-world laboratories: An experience-based comparison. GAIA, 27 (S1), (S. 52-59). (im Druck). 
Schäpke, N., Stelzer, F., Bergmann, M., Singer-Brodowski, M., Wanner, M., Caniglia, G., \& Lang, D. (2017). Reallabore im Kontext transformativer Forschung. Ansatzpunkte zur Konzeption und Einbettung in den internationalen Forschungsstand. (No. 1/2017). Leuphana Universität Lüneburg, Institut für Ethik und Transdisziplinäre Nachhaltigkeitsforschung.

Schneidewind, U., \& Singer-Brodowski, M. (2013). Transformative Wissenschaft: Klimawandel im deutschen Wissenschafts-und Hochschulsystem. Marburg: Metropolis.

Schneidewind, U., \& Singer-Brodowski, M. (2015). Vom experimentellen Lernen zum transformativen Experimentieren. Reallabore als Katalysator für eine lernende Gesellschaft auf dem Weg zu einer Nachhaltigen Entwicklung. Zeitschrift für Wirtschaftsund Unternehmensethik, 16 (1), (S. 10-23).

Seebacher, A., Alcántara, S., \& Quint, A. (2018a). Akteure in Reallaboren - Reallabore als Akteure. In R. Defila \& A. Di Giulio (Hrsg.), Transdisziplinär und transformativ forschen. Eine Methodensammlung (S. 155-159). Wiesbaden: Springer VS.

Seebacher, A., Alcántara, S., \& Quint, A. (2018b). Der Partizipationsmythos „Partizipation bedeutet, alle immer an allem zu beteiligen“. In R. Defila \& A. Di Giulio (Hrsg.), Transdisziplinär und transformativ forschen. Eine Methodensammlung (S. 101-104). Wiesbaden: Springer VS.

Singer-Brodowski, M., Beecroft, R., \& Parodi, O. (2018). Learning in real-world laboratories: A systematic impulse for discussion. GAIA, 27 (S1), (S. 23-27). (im Druck).

Trenks, H., Waitz, C., Meyer-Soylu, S., \& Parodi, O. (2018). Mit einer Realexperimentreihe Impulse für soziale Innovationen setzen - Realexperimente initiieren, begleiten und beforschen. In R. Defila \& A. Di Giulio (Hrsg.), Transdisziplinär und transformativ forschen. Eine Methodensammlung (S. 233-268). Wiesbaden: Springer VS.

Wagner, F., \& Grunwald, A. (2015). Reallabore als Forschungs-und Transformationsinstrument. Die Quadratur des hermeneutischen Zirkels. GAIA, 24 (1), (S. 26-31).

Waitz, C., Quint, A., Trenks, H., Parodi, O., Jäkel, A., Lezuo, D. \& Wäsche, H. (2018). Das Reallabor als Motor für nachhaltige Quartiersentwicklung - Erfahrungen aus dem Karlsruher Experimentierraum, Berichte. Geographie und Landeskunde, 91 (1), (S. 6780). (im Druck).

WBGU (Wissenschaftlicher Beirat für Globale Umweltveränderungen) (2011). Welt im Wandel. Gesellschaftsvertrag für eine Große Transformation. Hauptgutachten 2011. Berlin: WBGU. Verfügbar unter: http://www.wbgu.de/hauptgutachten/hg-2011-Trans formation. Zugegriffen am 18.02.2018.

Weichhart, P. (2008). Entwicklungslinien der Sozialgeographie. Von Hans Bobek bis Benno Werlen. Stuttgart: Steiner.

Wiek, A., Withycombe, L., \& Redman, C. L. (2011). Key competencies in sustainability. Sustainability Science, 6 (2), (S. 203-218). doi: 10.1007/s11625-011-0132-6. 
Open Access Dieses Kapitel wird unter der Creative Commons Namensnennung 4.0 International Lizenz (http://creativecommons.org/licenses/by/4.0/deed.de) veröffentlicht, welche die Nutzung, Vervielfältigung, Bearbeitung, Verbreitung und Wiedergabe in jeglichem Medium und Format erlaubt, sofern Sie den/die ursprünglichen Autor(en) und die Quelle ordnungsgemäß nennen, einen Link zur Creative Commons Lizenz beifügen und angeben, ob Änderungen vorgenommen wurden.

Die in diesem Kapitel enthaltenen Bilder und sonstiges Drittmaterial unterliegen ebenfalls der genannten Creative Commons Lizenz, sofern sich aus der Abbildungslegende nichts anderes ergibt. Sofern das betreffende Material nicht unter der genannten Creative Commons Lizenz steht und die betreffende Handlung nicht nach gesetzlichen Vorschriften erlaubt ist, ist für die oben aufgeführten Weiterverwendungen des Materials die Einwilligung des jeweiligen Rechteinhabers einzuholen. 